\title{
Deep Sequencing Uncovers Rice Long siRNAs and Its Involvement in Immunity Against Rhizoctonia solani
}

\author{
Dongdong Niu, Xin Zhang, Xiaoou Song, Zhihui Wang, Yanqiang Li, Lulu Qiao, Zhaoyun Wang, Junzhong Liu, Yiwen \\ Deng, Zuhua He, Donglei Yang, Renyi Liu, Yanli Wang, and Hongwei Zhao ${ }^{\dagger}$
}

First, second, third, fourth, sixth, seventh, and fourteenth authors: College of Plant Protection, Nanjing Agricultural University, Nanjing 210095, China; first, second, third, fourth, sixth, and fourteenth authors: Key Laboratory of Integrated Management of Crop Diseases and Pests (Nanjing Agricultural University), Ministry of Education; second author: Institute of Industrial Crops, Shanxi Academy of Agricultural Sciences, Taiyuan 030000, Shanxi, China; fifth and twelfth authors: Shanghai Center for Plant Stress Biology, Shanghai Institutes of Biological Sciences, Chinese Academy of Sciences, China; eighth, ninth, and tenth authors: National Key Laboratory of Plant Molecular Genetics, Shanghai Institute of Plant Physiology and Ecology, Chinese Academy of Sciences, Shanghai, China; eleventh author: College of Agriculture, Nanjing Agricultural University, Nanjing 210095, China; and thirteenth author: State Key Laboratory Breeding Base for Zhejiang Sustainable Pest and Disease Control, Institute of Plant Protection and Microbiology, Zhejiang Academy of Agricultural Sciences, Hangzhou, 310021, China.

Accepted for publication 1 September 2017.

\begin{abstract}
Small RNA (sRNA) is a class of noncoding RNA that can silence the expression of target genes. In rice, the majority of characterized sRNAs are within the range of 21 to 24 nucleotides (nt) long, whose biogenesis and function are associated with a specific sets of components, such as Dicerlike (OsDCLs) and Argonaute proteins (OsAGOs). Rice sRNAs longer than $24 \mathrm{nt}$ are occasionally reported, with biogenesis and functional mechanism uninvestigated, especially in a context of defense responses against pathogen infection. By using deep sequencing, we identified a group of rice long small interfering RNAs (lsiRNAs) that are within the range of 25 to $40 \mathrm{nt}$ in length.

Our results show that some rice lsiRNAs are differentially expressed upon infection of Rhizoctonia solani, the causal agent of the rice sheath blight disease. Bioinformatic analysis and experimental validation indicate that some rice lsiRNAs can target defense-related genes. We further demonstrate that rice lsiRNAs are neither derived from RNA degradation nor originated as secondary small interfering RNAs (siRNAs). Moreover, lsiRNAs require OsDCL4 for biogenesis and $O s A G O 18$ for function. Therefore, our study indicates that rice lsiRNAs are a unique class of endogenous sRNAs produced in rice, which may participate in response against pathogens.
\end{abstract}

Small RNA (sRNA) is a class of 21 to 24 nucleotide (nt)-long RNA molecules that can specifically target genes with reverse complementary sequences and mediate gene silencing. sRNAs regulate the expression of target genes either through transcriptional gene silencing (TGS) or posttranscriptional gene silencing (PTGS) (Baulcombe 2004). Plant sRNAs play important roles in processes such as leaf morphogenesis, floral organ identity, hormone signaling, nutrient transportation, assimilation and stress responses (Ambros and Chen 2007; Jones-Rhoades et al. 2006; Rogers and Chen 2013; Voinnet 2009). Plant sRNAs are classified into two subgroups, microRNAs (miRNAs) and short interfering RNAs (siRNAs), according to their biogenesis and precursor structures (Chen 2009). siRNAs can be further divided into heterochromatic siRNAs (hc-siRNAs), transacting siRNAs (ta-siRNAs), long siRNAs (lsiRNAs), and natural antisense transcripts-derived siRNAs (nat-siRNAs) (Chapman and Carrington 2007; Jin and Zhu 2010; Katiyar-Agarwal and Jin 2010). In plants, the functions of sRNAs depend on several conserved protein families, such as Dicer-like proteins (DCL), Argonaute (AGO) proteins, RNA-dependent RNA polymerases (RDRs), and the sRNA methyltransferase (HEN1) (Chapman and Carrington 2007).

Plant sRNAs are important players in defense response against pathogens (Duan et al. 2012; Katiyar-Agarwal and Jin 2010; Pumplin

†Corresponding author: H. Zhao; E-mail: hzhao@njau.edu.cn

First and second author contributed equally to this work.

*The $\boldsymbol{e}$-Xtra logo stands for "electronic extra" and indicates that five supplementary figures and three supplementary tables are published online.

(c) 2018 The American Phytopathological Society and Voinnet 2013). miR393 is the first identified defense-related sRNA that can be induced by pathogen associated molecular pattern (PAMP) molecules such as flg22. By silencing an auxin receptor, miR393 efficiently suppresses auxin signaling and positively contributes to the PAMP triggered immunity (PTI) (Navarro et al. 2006). Interestingly, the miRNA originated from another strand of the miR393 duplex, miR393*, can be activated by the effector triggered immunity (ETI) signaling. miR393*can influence exocytosis of some pathogenesis-related protein (PRs) and contributes to plant defense against bacterial pathogens (Zhang et al. 2011). The functionally cognate miR393/miR393b* pair demonstrates that both strands of an miRNA duplex (previously considered as miRNA and its passenger miRNA without function) are biologically related and functionally coregulated. It suggests that plant immunity system can adjust PTI and ETI machineries by such a pair of miRNAs from a single transcript, more efficiently and effectively. Similar phenomenon was found on Arabidopsis miR825/825*, both of which are reduced by a Bacillus cereus strain and contribute to induced systemic resistance (ISR) (Niu et al. 2016). Several other sRNAs involved in plant defense have also been identified, including their mechanism of action through TGS or PTGS (Niu et al. 2015; Seo et al. 2013).

In crops and vegetables, endogenous plant sRNAs are also critical factors in shaping the relationship with their associated pathogens. This is demonstrated by observations made in many species such as rice, wheat, tomato, and citrus. For example, Zhai and colleagues identified several 22-nt miRNAs that target conserved domains of nucleotide-binding, leucine-rich repeats (NB-LRRs) genes in Medicago and potato, which trigger the production of phased, secondary siRNAs (phasiRNAs) at the cleavage site. These transacting siRNAs can target other NB-LRRs genes and affect plant immunity (Zhai et al. 2011). In wheat, diverse set of miRNAs are identified in response to powdery 
mildew infection (Xin et al. 2010). In citrus, miR399 is induced by the Huanglongbing (HLB) bacteria. The expression of citrus miR399 improves the inorganic phosphorus deficiency (one of the HLB disease symptoms) through elevating phosphorus transporter activity, which alleviates HLB symptoms (Zhao et al. 2013). When infected by Fusarium oxysporum, expression of two tomato miRNAs which are capable of targeting genes encoding proteins with full or partial nucleotide-binding (NB) domains are suppressed. Silencing of these target genes leads to a resistance-to-susceptibility switch phenotype in resistant cultivars (Ouyang et al. 2014). In rice, miR160 or miR398 are differentially expressed between resistant and susceptible cultivars after Magnaporthe oryzae challenge. Overexpression of miR160 or miR398 in a susceptible rice cultivar leads to enhance disease resistance toward $M$. oryzae, confirming that these two miRNAs are positive regulators of defense against the blast disease (Li et al. 2014). Taken together, multiple evidence supports the idea that endogenous sRNAs are critical players of plant innate immunity, and their study will be of great significance on plant disease control and management.

Although most of the functional sRNAs studied to date are 21 to $24 \mathrm{nt}$ long, sRNAs with length more than $24 \mathrm{nt}$ have been reported in different species sporadically. The 27- to 30-nt small scan RNAs (scnRNAs) associated with Argonaute proteins have been shown to act in the DNA elimination process in Tetrahymena (Hock and Meister 2008). PIWI-interacting RNAs (piRNAs), which are generally 24 to $32 \mathrm{nt}$ in length, bind specifically to the PIWI subfamily of Argonaute proteins and function in germline stem-cell maintenance and selfrenewal (Lin and Spradling 1997). Carone et al. (2009) reported the 35 to 42 nt sRNA (centromere repeat associated short interacting RNAs; crasiRNAs), which is produced from transcription of repeat regions and is proposed to be essential components of cellular stability and chromosome segregation in tammar wallaby (Macropus eugenii) (Brown and O'Neill 2010; Carone et al. 2009). In plants, a novel class of endogenous long siRNAs (lsiRNAs; 30 to $40 \mathrm{nt}$ ) were found to be induced by bacterial infection or specific growth conditions, such as cell suspension culture (Katiyar-Agarwal et al. 2007). For example, AtlsiRNAl is a cluster of heterogeneous lsiRNAs, most being 39 to 41 $\mathrm{nt}$ in length, is strongly induced by Pseudomonas syringae pv. tomato containing a Rpt2 avirulent factor ( $P$. syringae pv. tomato [avrRpt2]). AtlsiRNA1 silences AtRAP is a negative regulator of plant defense. When AtRAP was knocked out, increased resistance to both virulent and avirulent strains was observed in Arabidopsis, indicating defense against bacterial pathogens could be enhanced through upregulation of AtlsiRNA1 (Katiyar-Agarwal et al. 2007). Long nonprotein coding RNAs (npcRNAs) responsive to powdery mildew infection were identified in wheat. The authors found that wheat long npcRNAs showed tissue dependent expression patterns and were responsive to powdery mildew infection and heat stress (Xin et al. 2010). siRNAs independent of DCLs (sidRNAs; 20 to $60 \mathrm{nt}$ in length) that are associated with AGO4 and capable of directing DNA methylation have also been reported. (Ye et al. 2016). In rice, a new class of miRNAs called long miRNAs (lmiRNAs) that are $24 \mathrm{nt}$ long and are loaded into AGO4 clade proteins have been identified, which direct DNA methylation both in cis and trans (Wu et al. 2010). This evidence indicates that a variety of sRNAs longer than 24 nt exist in vivo and are functional. It is of great interest to investigate whether these long sRNAs are associated with any unknown biogenesis or functional characteristics.

Rice is one of the major food crops in the world, especially in Asia. Rice sheath blight, caused by Rhizoctonia solani, is considered as one of the major diseases that lead to significant losses of yields and quality each year (Lee and Rush 1983). Here, we used a deep sequencing approach to investigate the global expression of endogenous long siRNAs in rice against $R$. solani infection. We identified a new class of 25- to 40-nt-long rice endogenous lsiRNAs (osalsiRNAs) whose expression is specifically induced by $R$. solani infection. Our results indicate that $R$. solani specifically induces the expression of several osa-lsiRNAs, whose target genes include some defense-related genes. $R$. solani infection is facilitated by inhibited expression of defense-related genes through osa-lsiRNAs-mediated gene silencing, indicating a crucial role of osa-lsiRNAs in shaping the relationship between rice and $R$. solani.

\section{MATERIALS AND METHODS}

Pathogen infection and sRNA library construction. Rice (Oryza sativa L. japonica. 'Nipponbare') was grown in soil in a growth room maintained at $26^{\circ} \mathrm{C}$ and $70 \%$ relative humidity with a $12 / 12$-h day $\left(200 \mu \mathrm{E} \mathrm{m}^{-2} \mathrm{~s}^{-1}\right) /$ night. The rice sheath blight disease pathogen $R$. solani AG1 IA was used in this study. The fungal strains were grown on potato dextrose agar (PDA: $200 \mathrm{~g}$ of potato, $20 \mathrm{~g}$ of dextrose, and $20 \mathrm{~g}$ of agar) at $28^{\circ} \mathrm{C}$. For inoculation, four-leaf-stage seedlings were covered by a mycelium plug $(5 \mathrm{~mm}$ in diameter) of actively growing $R$. solani mycelium onto the stem. The samples were collected at $0,24,48$, and $72 \mathrm{~h}$ postinoculation (hpi).

RNA extraction and library construction was carried out as described previously (Katiyar-Agarwal et al. 2007; Padmanabhan et al. 2009). Briefly, total RNA was isolated from infiltrated leaves and removed large ribosomal RNA (rRNA) from total RNA by RiboMinus Plant Kit as describe (Invitrogen, A10838-08). Then the RiboMinus RNA was fractionated on $15 \%$ denaturing polyacrylamide gel. RNA molecules ranging from 20 to $45 \mathrm{nt}$ were excised and ligated to $5^{\prime}$ - and 3'-RNA adaptors using T4 RNA ligase followed by RT-PCR and gel purification as described in the Instruction from Illumina Inc. The sRNA libraries were sequenced by Illumina Inc. and UCR core facility. The 20 to $24 \mathrm{nt}$ will be reported in a separate manuscript.

Long siRNAs analysis. Raw reads were processed to trim the adaptors using cutadapt. After adaptor sequence was removed, clean reads of 25 to $40 \mathrm{nt}$ length were clustered into unique reads. The reads that match known rice repeats, rRNAs, tRNAs, snRNAs, and snoRNAs (allow 2 mismatches) were removed, and remaining reads were further mapped to the rice genome (version 6.1) using bowtie. The expression level of sRNAs were normalized to the all mapped reads using TPTM (transcripts per 10 million). Audic and Claverie method were used for analyzing differentially expression of sRNAs with a significant threshold $(P \leq 0.01)$ as previously described. Bonferroni method was used to adjust $P$ values for multiple comparisons. The psTarget program was used to predict the target of long sRNAs. A penalty scoring system ( 0 to 5 ) was used for sRNA target prediction, in which score 0 is the best (perfect match). The penalty score for a mismatch or gap in sRNA and target alignment is 1 , or 1.5 when it is located in the seed region ( 2 to $13 \mathrm{nt}$ ); the penalty score for a G:U pair is 0.5 . The $\mathrm{R}$ package TopGO were used for target gene GO term enrichment analysis as described before. Adrian Alexi's improved weighted scoring algorithm and Fisher's test was used to determine the significance of GO term enrichment. Significantly enriched GO terms were identified as those with a $P$ value less than 0.05 .

Northern blot analysis. RNA-blot analyses for long siRNAs from total extract were performed as described previously (KatiyarAgarwal et al. 2007). Total RNA was extracted using TRIzol Reagent (Invitrogen) following the manufacturer's instructions. RNA is resolved on a 17\% denaturing $8 \mathrm{M}$ urea-PAGE gel and then transferred and chemically crosslinked onto a Hybond $\mathrm{N}^{+}$membrane (GE Healthcare Life Sciences) using N-(3-dimethylaminopropyl)$\mathrm{N}^{\prime}$-ethylcarbodiimide hydrochloride. lsiRNAs were detected using end-labeled oligonucleotide probes and exposed to a phosphor imager screen. Expression level was quantified by using Image $\mathbf{J}$ as instructed.

qRT-PCR. The total RNA used for qRT-PCR was from the same set of RNA used for RNA-blot analyses. To detect the quality and quantity of RNA, a NanoDrop-1000 was accessed (NanoDrop). The RNA was then reverse transcripted into cDNA by using PrimeScript RT Reagent Kit (Takara, Japan). The qRT-PCR was performed in $15 \mu \mathrm{l}$ of reaction mixture consisting $1.5 \mu \mathrm{l}$ of $1 \times$ SYBR Green (Invitrogen), $1.5 \mu \mathrm{l}$ of PCR buffer, $0.3 \mu \mathrm{l}$ of $10 \mathrm{mM}$ DNTPs (Takara), $0.3 \mu \mathrm{l}$ of Taq, $0.3 \mu \mathrm{l}$ of ROX DYE2 (Vazyme, China), $1.5 \mu \mathrm{l}$ of $2 \mathrm{mM}$ 
each primer and $2 \mu \mathrm{l}$ of appropriate diluted cDNA. The conditions for real-time RT-PCR were listed as follow: $94^{\circ} \mathrm{C}$ for $3 \mathrm{~min}$, then 40 cycles at $94^{\circ} \mathrm{C}$ for $30 \mathrm{~s}$ and $58^{\circ} \mathrm{C}$ for $30 \mathrm{~s}$ followed by $72^{\circ} \mathrm{C}$ for $35 \mathrm{~s}$ for PCR amplification. Transcript levels of each gene were measured by the Applied Biosystems 7500 (Applied Biosystems) according to the manufacturer's instructions. 18s rRNA was used as a quantitative control in the qRT-PCR analysis.

Vector construction. The enzyme digestion connection method was used to make the long siRNAs artificial constructs by using WMD3 online (http://wmd3.weigelworld.org), and were cloned into the pEG202 vector. The homologous recombination method was used to make target genes constructs by using ClonExpress II One Step Cloning Kit (Vazyme), and were cloned into the pEG202 vector.

Protein extraction and analysis. Tissue sample was ground in liquid nitrogen and total proteins were extracted using $2 \times$ SDS loading buffer. The samples were resolved on a 12\% SDS-PAGE gel and transferred onto an Amersham Hybond-P PVDF membrane (GE Healthcare) using a Tris-Glycine transfer buffer. Membranes were blocked with 5\% milk in TBS-Tween-20 0.05\% (TBST) for $40 \mathrm{~min}$ and incubated overnight at $4{ }^{\circ} \mathrm{C}$ with 1:5,000 dilutions of primary antibodies mouse anti-Flag conjugated to horseradish peroxidase (Abmart), and washed three times with TBST, then incubated overnight at $4^{\circ} \mathrm{C}$ with $1: 1,000$ dilutions of secondary antibodies (Beytime). Membranes were washed three times with TBST. Detection was performed using the ECL Plus Western Blotting Detection Reagents (GE Healthcare) and ChemiDoc Touch Imager (Bio-Rad). Image data were analyzed with Image Lab Software (Bio-Rad) and assembled in Adobe Photoshop CS6.

Transient expression analysis in $N$. Benthamiana. Transient co-expression assays in $N$. benthamiana were performed by infiltrating 3-week-old $N$. benthamiana plants with Agrobacterium GV3101 $\left(\mathrm{OD}_{600}\right.$ [optical density at $600 \mathrm{~nm}$ ] $\left.=1.0\right)$ harboring constructs containing the long siRNAs (pEG202) with Agrobacterium GV3101 containing $\left(\mathrm{OD}_{600}=1.0\right)$ target genes CDS (pEG202), and miR319b (pEG202) was a control. Leaf tissue was collected at 48 hpi. Protein expression was detected by western blot.

Statistical analysis. All data obtained were subjected to twoway analysis of variance (ANOVA) and a Student's $t$ test was used to determine significant differences between the treatments. The comparisons with $* * P<0.01$ and $* P<0.05$ were considered significantly different.

\section{RESULTS}

Characterization of osa-lsiRNAs by deep-sequencing analysis. To investigate the potential roles that lsiRNAs may play in rice defense against $R$. solani, osa-lsiRNAs expression profiles between rice (japonica cultivar Nipponbare) infected with $R$. solani (strain AG1-IA) and noninfected rice (mock) were compared and analyzed. Due to the quick responding nature of plant innate immunity to $R$. solani infection, osa-lsiRNAs libraries were constructed with stem at $24 \mathrm{~h}$ postinoculation (hpi; RS-24). At 48 hpi, a large number of hyphae were produced at inoculation site and the rice stalk gradually became yellow and lodging (Supplementary Fig. S1). Long siRNAs fractions ranging from 20 to $45 \mathrm{nt}$ were

TABLE 1. Summary of osa-lsiRNAs in Rhizoctonia solani-infected samples

\begin{tabular}{lcccc}
\hline Read categories & Mock & $(\%)$ & Infected & $(\%)$ \\
\hline $\begin{array}{l}\text { Total reads } \\
\begin{array}{l}\text { Rice reads } \\
\text { (mapped to rice }\end{array}\end{array}$ & $59,304,915$ & 100.00 & $45,418,644$ & 100.00 \\
$\quad$ genome) & $45,229,961$ & 76.27 & $29,210,015$ & 64.31 \\
$\begin{array}{l}\text { Aligned to Rfam } \\
\quad \text { (rRNA, tRNA, snRNA, }\end{array}$ & & & & \\
$\quad$ and snoRNA) & $16,804,127$ & 28.34 & $15,930,982$ & 35.08 \\
Rice sRNA (total reads) & $28,425,834$ & 47.93 & $13,279,033$ & 29.24 \\
Rice sRNA (unique reads) & $2,267,454$ & $\ldots$ & $1,069,396$ & $\ldots$ \\
\hline
\end{tabular}

cloned and sequenced by Illumina HiSeq platform. A total of $59,304,915$ and 45,418,644 reads were generated from the control (control-24) and treatment (RS-24) groups, respectively (Table 1). These reads were mapped to the rice genome (Rice Genome Annotation Project), which resulted in 45,229,961 and 29,210,015 rice reads (corresponding to 76.27 and $64.31 \%$ of the total reads), respectively. Reads were further searched against the Rfam database (http://xfam.org/) to remove known sRNAs such as ribosomal RNA (rRNA), transfer RNA (tRNA), and small nucleolar RNAs (snoRNAs). A total of 28,425,834 and 13,279,033 reads passed the aforementioned criteria and were obtained from the mock and infected group, respectively, corresponding to 2,267,454 and $1,069,396$ unique osa-lsiRNAs. These identified osa-lsiRNAs were regarded as osa-lsiRNAs and were subjected to further analysis (Table 1).

Among the osa-lsiRNAs identified, lengths of 28 and 29 nt were the most abundant ones, each accounting for more than $10 \%$ of the total reads (Fig. 1A). The $27 \mathrm{nt}$ and 30- to 36-nt-long siRNAs were between 5 and $10 \%$ of total reads. Collectively, osa-lsiRNAs from 27 to $36 \mathrm{nt}$ accounted for more than $85 \%$ of the total reads we identified in this research, while the remaining populations represented less than $15 \%$ of the total reads (Fig. 1A). Abundance of osa-lsiRNAs longer than $37 \mathrm{nt}$ dropped rapidly to less than $1 \%$, indicating that lengths between 25 and 40 nt should cover most of the long siRNA populations.

Regardless of the varied length, four different types of nucleotide (A, U, G, and C) displayed similar frequency on the first nucleotide position (Fig. 1B), with guanine (G; 28.36\%) a little bit higher presented and uracil (U) relatively less presented $(20.95 \%)$ at the 5 '-most position of the osa-lsiRNAs. The other two types of nucleotides, adenine (A) and cytosine (C), represented 27.59 and $23.10 \%$ of the total population, respectively. Osa-lsiRNAs were further analyzed according to their origination on the rice genome. Most of the osa-lsiRNAs (91.32\%) were derived from transposable elements and repeat regions (TE and repeat); $3.86 \%$ were derived from intergenic region; $2.21 \%$ were derived from promoter region; and only $0.12 \%$ were derived from miRNA region. Osa-lsiRNAs originating from coding regions were also identified, most of which $(2.24 \%)$ generated from the sense orientation, with only a small fraction from the antisense orientation (Fig. 1C).

Identification of osa-IsiRNAs responsive to $R$. solani infection. Among millions of osa-lsiRNAs identified, we focused on osa-lsiRNAs that are induced or reduced more than twofold $\left(\mid \log _{2}[\right.$ treated/mock $] \mid \geq 1$ ) (Supplementary Table S1). Bioinformatics analysis revealed that 14,415 reads fitted this criterion, implying that a big panel of osa-lsiRNAs are differentially expressed after $R$. solani infection. We hypothesized that if an osa-lsiRNA is involved in rice defense against $R$. solani infection, this specific candidate should fulfill one or more following pre-requirements: (i) candidates should not be expressed in very low level; (ii) candidates should show significantly varied expression after $R$. solani infection; (iii) candidates should target some resistance-related genes; and (iv) candidate sequence should demonstrate a relatively strict match to their target genes. To evaluate this hypothesis, we applied more stringent criteria (including the following: reads [in either treatment] $\geq 60$; [treated $/ \mathrm{mock}] \geq 3$ or [treated $/$ mock] $\leq$ 0.33 ; target genes with defense-related functions; matching score $\leq 3$ ) that would effectively eliminate potential false positive candidates. By applying these criteria, we identified 14 differentially expressed osa-lsiRNAs, which ranged from 25 to $30 \mathrm{nt}$ in length, and whose target genes are involved in a variety of functions such as disease resistance related factors, kinase, transcription factors, and signaling components (Table 2).

The differential expression pattern was further validated by using northern blotting, in which the U6 small nuclear RNA (U6) was used as a loading control. Due to low in vivo abundance, many of these candidates could not be detected even after prolonged exposure $(3 \times$ longer exposure than regular). At the end, we were able 
to validate six osa-lsiRNAs with clear northern results (Fig. 2A; Supplementary Fig. S2). We further focused on the osa-lsiRNAs whose expression signal were sufficient for Image $\mathbf{J}$ quantification (Fig. 2A). Osa-lsiR51031 is a 30-nt lsiRNA, whose expression was suppressed by $R$. solani infection. At as early as $24 \mathrm{hpi}$, its relative abundance (RA) was about $50 \%$ of that at 0 hpi (RA $=[\text { Exp }]^{24}$ hpi/ Exp ${ }^{0 \mathrm{hpi}}$, and continued to 48 and $72 \mathrm{hpi}$, respectively. After 3 days of infection, the expression level of osa-lsiR51031 was almost below detectable level $(\mathrm{RA}=0.06)$ (Fig. 2A). In contrast, osa-lsiR118183, 194568 , and 73750 showed a sustained increase with $R$. solani infection (Fig. 2A). Osa-lsiR118183 was rapidly induced as early as
24 hpi $(\mathrm{RA}=3.1)$, and increased steadily to a medium time point (48 hpi; RA = 3.7) and a late time point $(72 \mathrm{hpi}$; RA $=4.0)$. Osa-lsiR73750 showed a delayed but stronger induced expression tendency, which showed no increase at 24 hpi but accumulated to more than 7 folds at $48 \mathrm{hpi}$; after that, it dropped to 3.2 folds at $72 \mathrm{hpi}$. Osa-lsiR194568 was under detectable level without $R$. solani infection. After infection, it demonstrated mild increase at 24 and $48 \mathrm{hpi}$ but increased quickly to more than 10 folds at $72 \mathrm{hpi}$ (Fig. 2A). Taken together, our results confirmed that some osa-lsiRNAs could be differentially expressed upon $R$. solani infection. This prompted us to further investigate the biological function of these lsiRNAs.
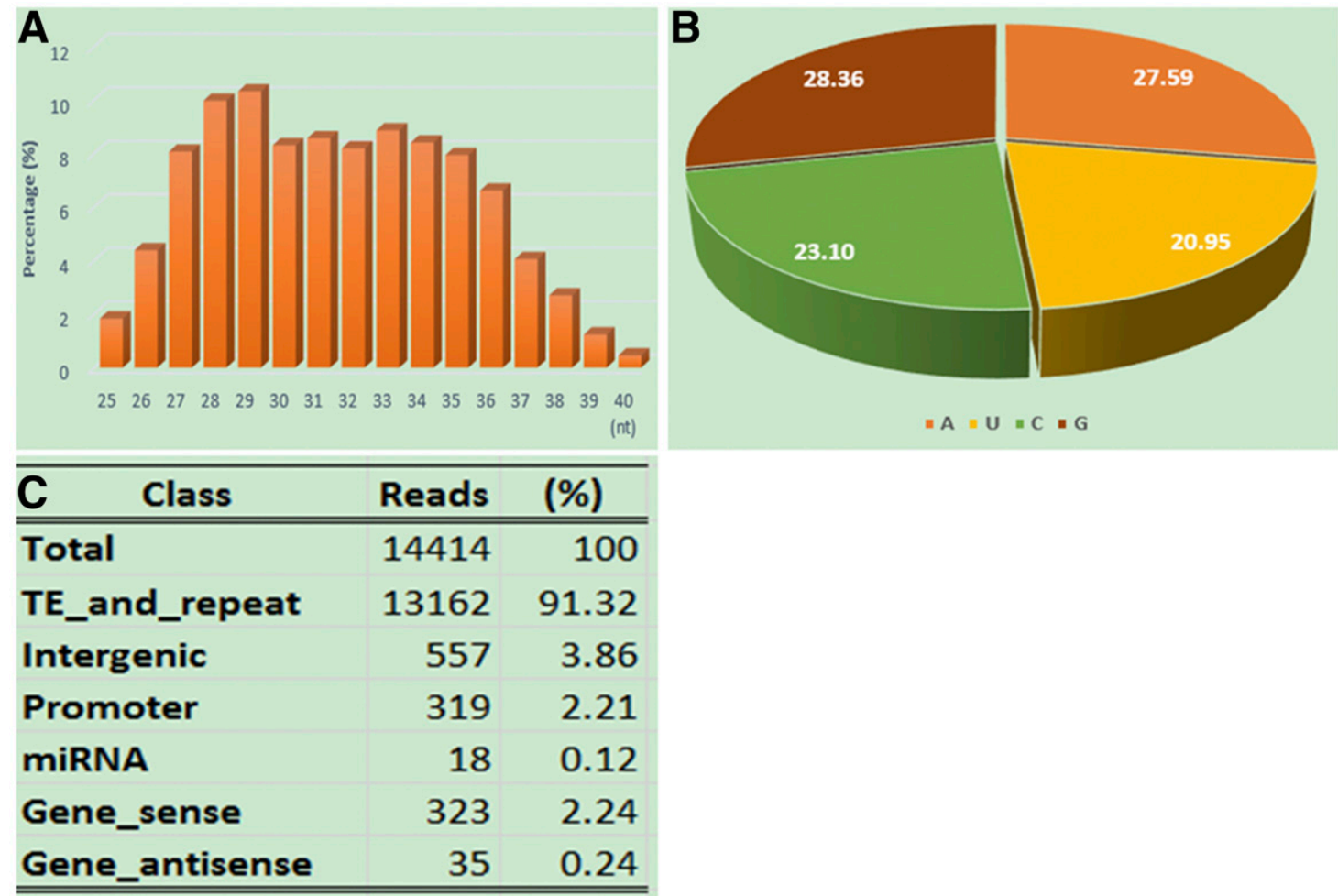

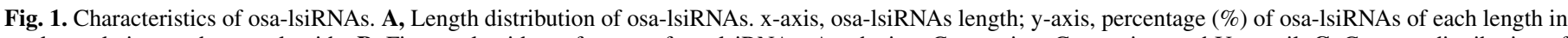

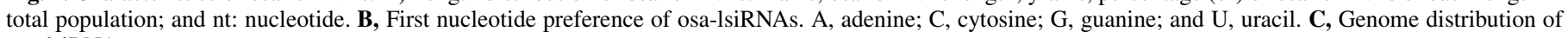
osa-lsiRNAs.

TABLE 2. Differentially expressed rice lsiRNAs and their potential target genes

\begin{tabular}{|c|c|c|c|c|c|}
\hline ID & Length (nt) & Target gene & Annotation & Score & Alignment \\
\hline lsiR118183 & 26 & Os08g06220 & Transferase & 0 & 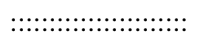 \\
\hline \multirow[t]{2}{*}{ 1siR51031 } & 30 & Os08g15322 & Cytochrome b559 subunit alpha & 0 & ::::::::::::::::::::::::: \\
\hline & & Os03g38930 & Expressed protein & 3 & 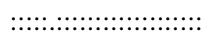 \\
\hline 1siR194568 & 25 & Os06g38990 & Receptor-like protein kinase 2 precursor & 2.5 & 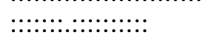 \\
\hline \multirow[t]{2}{*}{ lsiR73750 } & 29 & Os06g39780 & Expressed protein & 2.5 & 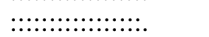 \\
\hline & & Os09g14490 & TIR-NBS type disease resistance protein & 2.5 & :::::::::.:::::::::: \\
\hline 1siR4052 & 30 & Os $11 \mathrm{~g} 41820$ & RNA recognition motif containing protein & 2.5 & 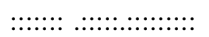 \\
\hline lsiR301048 & 29 & Os02g27620 & $\begin{array}{l}\text { Endonuclease/exonuclease/phosphatase } \\
\text { family domain containing protein }\end{array}$ & 2.5 & 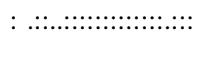 \\
\hline 1siR267188 & 29 & Os06g 12050 & mTERF family protein & 2.5 & ::.:.:.:.:.:.:.:.:.:.: \\
\hline \multirow[t]{2}{*}{ 1siR148949 } & 30 & Os06g 12050 & mTERF family protein & 3 & :.:.:.:.:.:.:.:.:.:.:.: \\
\hline & & Os $12 \mathrm{~g} 37860$ & Expressed protein & 3 & 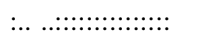 \\
\hline 1siR32311 & 29 & Os $12 g 02540$ & $\begin{array}{l}\text { Broad Complex BTB domain with } \\
\text { nonphototropic hypocotyl } 3 \text { NPH3 } \\
\text { and coiled-coil domains }\end{array}$ & 0 & 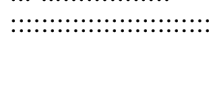 \\
\hline \multirow[t]{2}{*}{ 1siR218517 } & 29 & Os11g47030 & Receptor protein kinase & 3 & 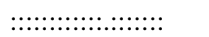 \\
\hline & & Os11g47240 & $\begin{array}{l}\text { Leucine-rich repeat receptor protein kinase } \\
\text { EXS precursor }\end{array}$ & 3 & 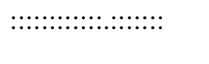 \\
\hline 1siR407572 & 30 & Os01g64790 & AP2 domain containing protein & 1.5 & 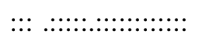 \\
\hline lsiR33332 & 28 & Os01g26160 & Exportin 1 & 3 & :::::::::::::::::::: \\
\hline 1siR407009 & 29 & Os01g04920 & $\begin{array}{l}\text { Glycosyl transferase, group } 1 \text { domain } \\
\text { containing protein }\end{array}$ & 3 & 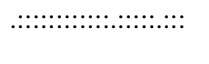 \\
\hline 1siR273010 & 29 & Os01g27150 & Cullin & 3 & 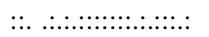 \\
\hline
\end{tabular}


For the four osa-lsiRNAs differentially expressed in $R$. solaniinfected rice plants, six target genes were identified by bioinformatics prediction. As summarized in Table 2, lsiR194568 targets a gene encoding a putative receptor-like protein kinase 2 precursor (Os06g38990; score = 3). Osa-lsiR118183 targets a gene encoding a putative transferase (Os08g06220), between which a $100 \%$ match was observed. Osa-lsiR51031 targets a gene encoding the putative cytochrome b559 subunit alpha (Os08g15322), between which a $100 \%$ match was observed as well. Osa-lsiR51031 also targets a gene encoding an expressed protein (OsO3g38930). Osa-lsiR73750 targets two genes (OsO6g39780 and OsO9g14490). Os06g39780 encodes an expressed protein with unknown function, while
Os09g14490 encodes a TIR-NBS type disease resistance protein. To exclude the possibility that the variated expression of these lsiRNAs target genes are due to simultaneously regulation by other differentially expressed regular sRNA, we searched a 20 to $24 \mathrm{nt}$ sRNAs sequencing database from rice either with or without $R$. solani infection (unpublished data). We did not find any potential dual-targeting events on these target genes described here. Therefore, we are confident to conclude that the observed correlation between lsiRNAs and their target genes is due to the regulatory role of lsiRNAs. We further examined the in vivo expression of each predicted target genes upon $R$. solani infection. The RNA used for qRTPCR was from the same set RNA for RNA-blot analyses. Os $08 g 15322$
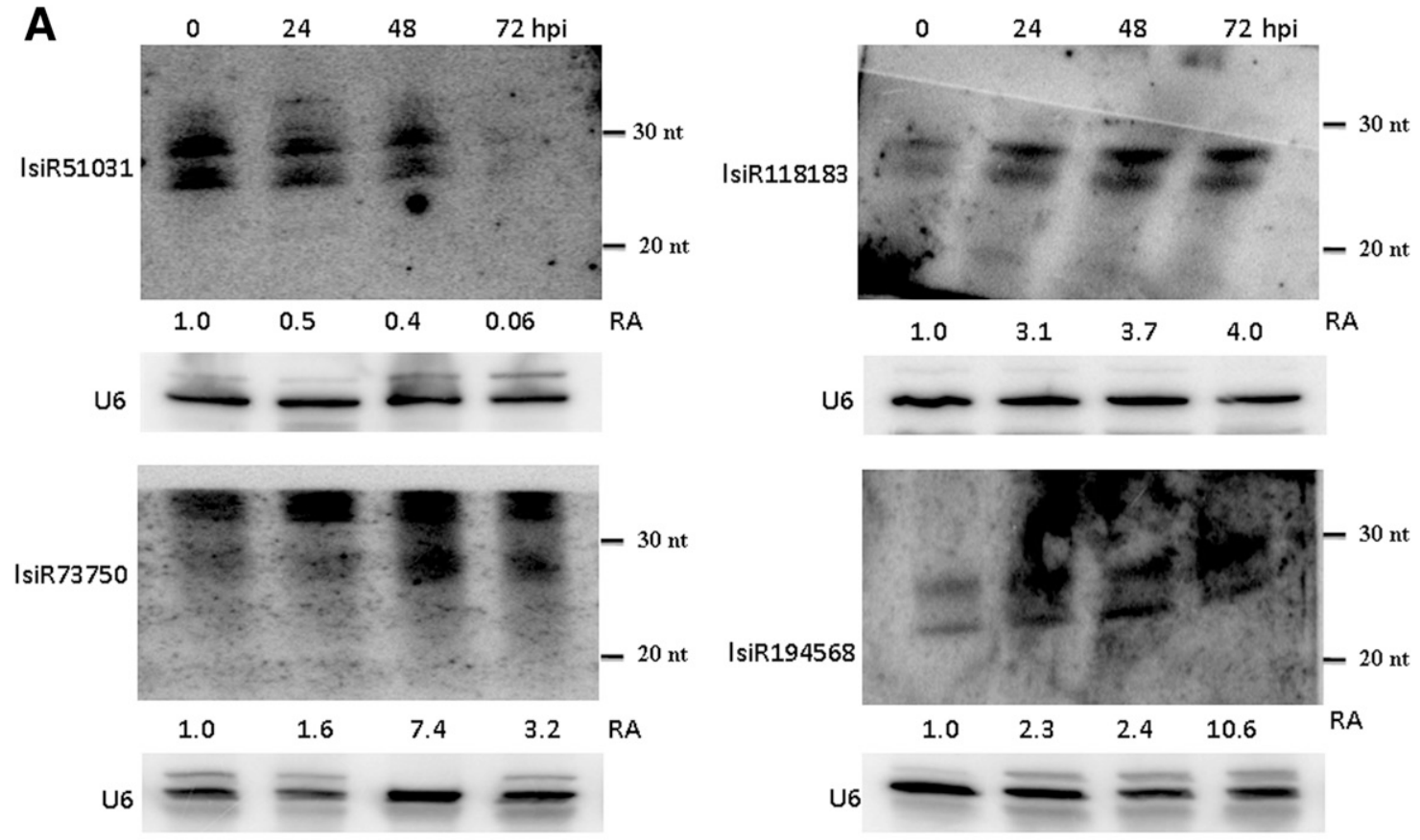

B
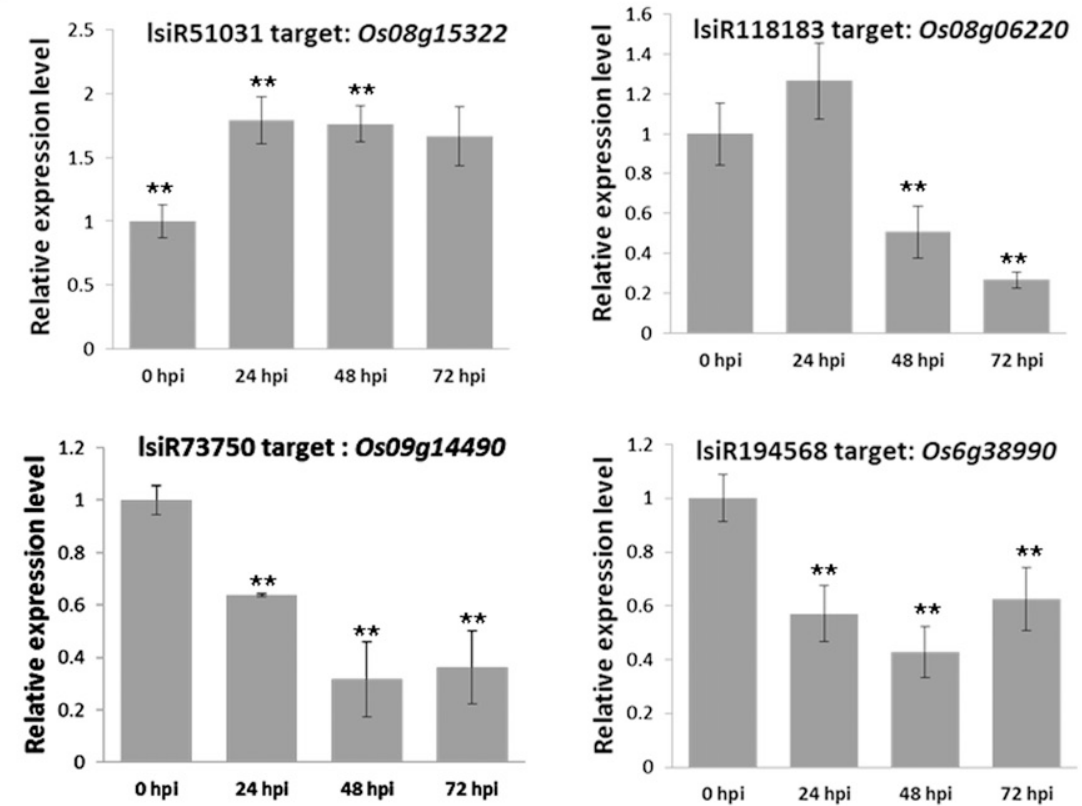

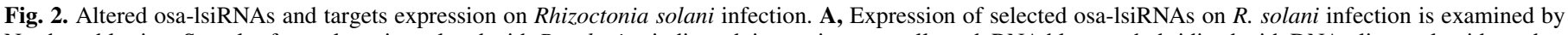

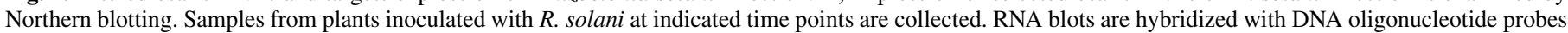

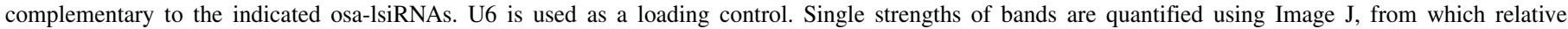

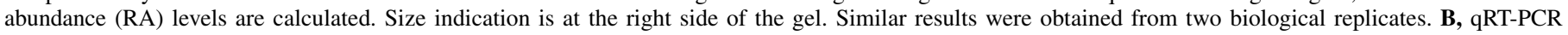

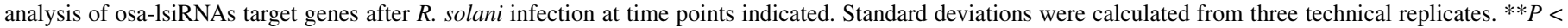
0.01 (Student's $t$ test). Similar results were obtained from three biological replicates. 
showed 1.8-fold increase since $24 \mathrm{hpi}$, which continued until $72 \mathrm{hpi}$. The increased expression of $O s 08 g 15322$ is cognately inverse to the expression pattern of siR51031, further suggesting a regulatory relationship between these two parties. Os09g 14490 showed a noticeable reduction since $24 \mathrm{hpi}$ and reached less than $40 \%$ of the original level at $72 \mathrm{hpi}$, which corresponds to the overall increased expression tendency of osa-lsiR73750. The expression of $\mathrm{OsO} 8 \mathrm{gO6220}$ and Os06g38990 also showed a reversed pattern with their cognate lsiRNAs, osa-lsiR118183 and osa-lsiR194568, respectively (Fig. 2B). Taken together, our data indicate that these genes are authentic target genes of their cognate lsiRNAs.

To validate that the predicted genes are authentic targets of these osalsiRNAs, both the predicted target genes and their corresponding osalsiRNAs were co-expressed transiently in tobacco ( $N$. benthamiana). Os08g06220 is a predicted target gene of osa-lsiR 118183 with perfect reverse complementary matching sequence. When transiently expressed in tobacco, Os08g06220 showed obviously lower expression level in a co-infiltration assay with osa-lsiR118183, than it was expressed alone, or co-infiltrated with an unrelated miRNA (OsmiR319b). Similar examinations were performed on other lsiRNAs. In conclusion, we were able to show that $O s 08 g 06220$ is a target gene of osa-lsiR118183; OsO8g15322 is a target of osa-lsiR51031; OsO6g38990 is a target of osa-lsiR194568, and Os09g14490 is a target of osa-lsiR73750 (Fig. 3). In contrast, Os03g38930 did not appear to be targeted by osa-lsiR51031, and $O s 06 g 39780$ is not a target of osalsiR73750 either (data not shown). Therefore, in the following study, we focused on these four target genes Os08g06220, Os08g15322, Os06g38990, and Os09g14490.

Osa-IsiRNAs are produced and function through canonical sRNA silencing components. Whether osa-lsiRNAs share a common biogenesis route with canonical rice sRNAs (miRNA and siRNA within 21 to $24 \mathrm{nt}$ range) is not known yet. To investigate the essential components involved in osa-lsiRNAs biogenesis, we examined osa-lsiRNA expression level in different $d c l$ mutants available to us. Northern blotting results showed that expression of osa-lsiR51031 was significantly reduced in $d c l 4$ mutant, while hardly any noticeable change was observed in both wild type and $d c l 1$ and $d c l 3$ mutants (Fig. 4A). Likewise, although less abundant, osa-lsiR73750 and osa-lsiR118183 showed similar expression pattern as osa-lsiR51031, that is, comparable expression in wild type and $d c l l$, but measurably reduced level in $d c l 4$ mutant (Fig. 4A). Interestingly, osa-lsiR73750 showed slightly reduced expression in $\mathrm{dcl} 3$ mutant whereas osa-lsiR118183 remained unaffected. OsalsiR194568 was under detectable level, which agrees with our observation that osa-lsiR194568 gradually reached to a detectable level after $R$. solani infection (Fig. 2A). These results indicate that osa-lsiRNAs require $D C L 4$ but not $D C L 1$ for maturation, and $D C L 3$ is likely involved. We also examined the expression level of these target genes in $\mathrm{dcl}$ mutants. In agreement, Os08g15322, Os08g06220, Os09g14490, and Os06g38990 demonstrated uniformly increased expression level in the $d c l 4$ mutant, while only Os06g38990 showed a dependence on DCL1 (Fig. 4B). In summary, our results indicate that these four genes are authentic target genes of aforementioned osalsiRNAs, whose generation involves the DCLA or other dicing process.

Components involved in osa-lsiRNAs function were also investigated. We measured expression level of osa-lsiRNAs target genes in various Argonaute mutants available to us (osagold, osago2, osago3, osago11, osago12, osago14, osago15, and osagol8). The osago mutant information is shown in Supplementary Table S2. We also confirmed AGOs expression level in these mutants by qRTPCR (Supplementary Fig. S3). Os08g06220, which is a target gene of osa-lsiR118183, showed a two- and fourfold increase in osago2 and osago18, respectively (Fig. 5). Os06g38990 (target of osalsiR194568) exhibited similar expression pattern, with a 15- and
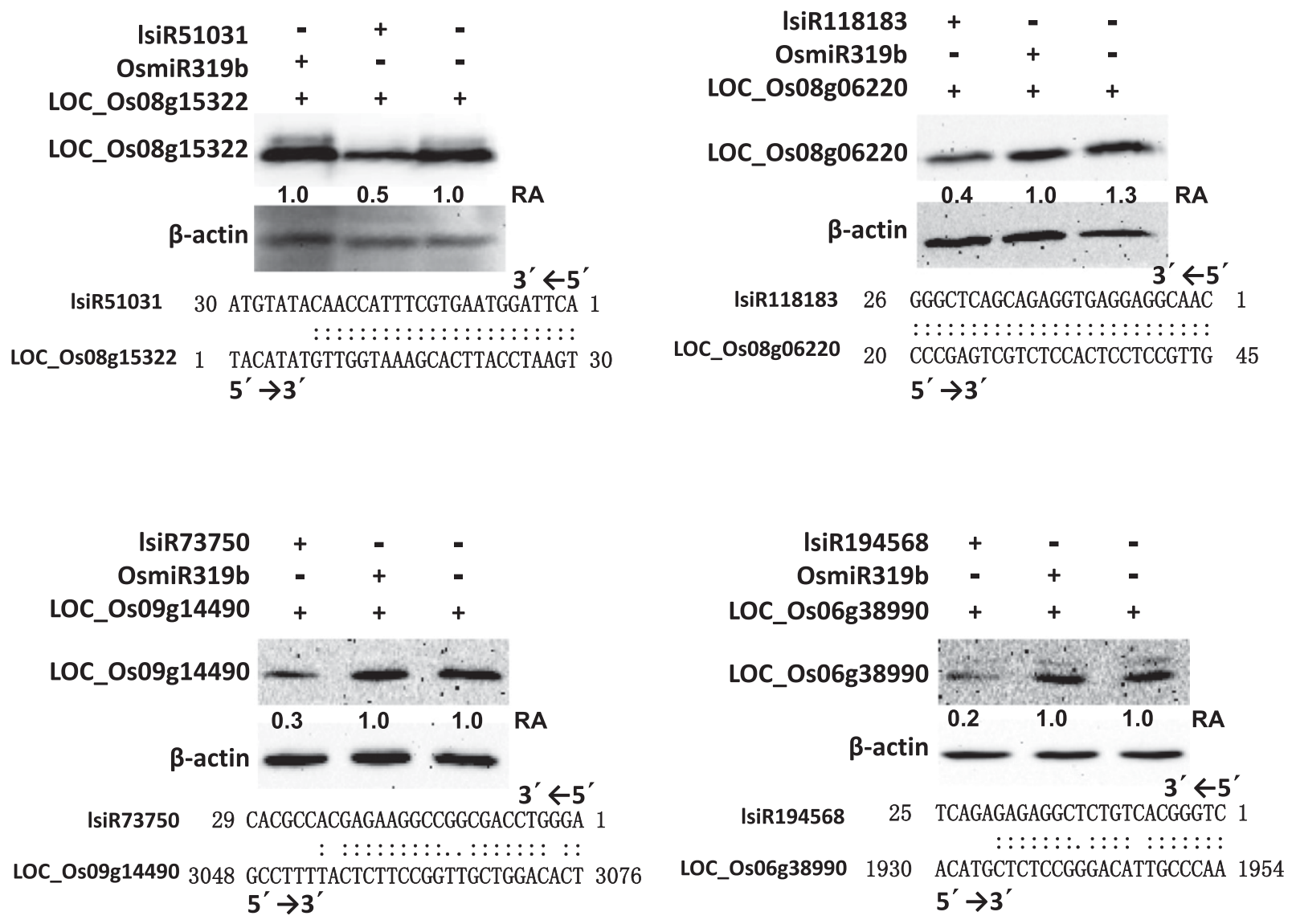

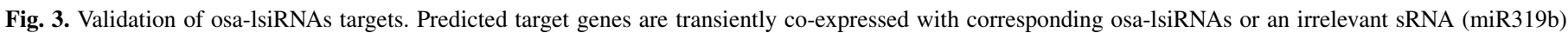

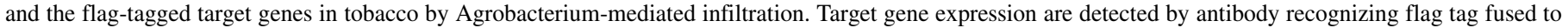
the target genes. $\beta$-actin was used as a loading control. Similar results were obtained from three biological replicates. 
12-fold increase in osago 2 and osago18, respectively. These results indicate that osa-lsiR118183 and osa-lsiR194568 may regulate the expression of its target genes through OsAGO18 and OsAGO2. Os09g14490 (target of osa-lsiR73750) and Os08g15322 (target of osa-lsiR51031) showed similar expression profiles among different osago mutants. In osago18, OsO9g14490 and OsO8g15322 were 4- and twofold increase, while $O s 09 g 14490$ showed a twofold increase, but the variation of $O \operatorname{SOSg} 15322$ was not significant. These results suggest that osa-1siR73750 and osa-lsiR51031 function depends on $O s A G O 18$ but may require OsAGO2. Taken together, our results suggest that, similar to canonical sRNAs, osa-lsiRNAs function through the AGO pathway. In specific, OsAGO18 and OsAGO2 are associated with osa-lsiRNAs function, whereas involvement of other $O s A G O s$ may be dispensable.

\section{DISCUSSION}

Plant sRNAs ranging from 21 to $24 \mathrm{nt}$ have received enormous attention for their biological function in plant growth, development, reproduction, and immunity. Generally, these sRNAs are divided into two categories: microRNA and siRNA, which differ both in term of biogenesis and function. Nevertheless, both canonical miRNA and siRNA are within 21 to 24 nt in length, and rely on Dicer-like and Argonaute proteins. sRNAs longer than 24 nt (lsiRNAs) have been identified in Arabidopsis, and implicated in plant resistance against bacterial pathogens (Katiyar-Agarwal et al. 2007). Whether these long siRNAs function in a unique way or contribute to a specific biological trait is unknown, especially in rice.

High-throughput sequencing has identified sRNAs at the wholegenome level in a context of plant-pathogen interaction (Naqvi et al. 2010; Niu et al. 2015; Seo et al. 2013). For rice endogenous sRNAs, Wu et al. (2015) characterized the sRNA profiles of rice plants infected by Rice dwarf virus (RDV) and Rice stripe virus (RSV) using high-throughput sequencing, and revealed a critical role of OsAGO18 in resistance against RSV. Guo et al. (2012) reported seven novel RSV-responsive miRNAs identified by highthroughput sequencing. A recent study identified 23 candidate rice miRNAs that may be involved in plant immunity against $R$. solani
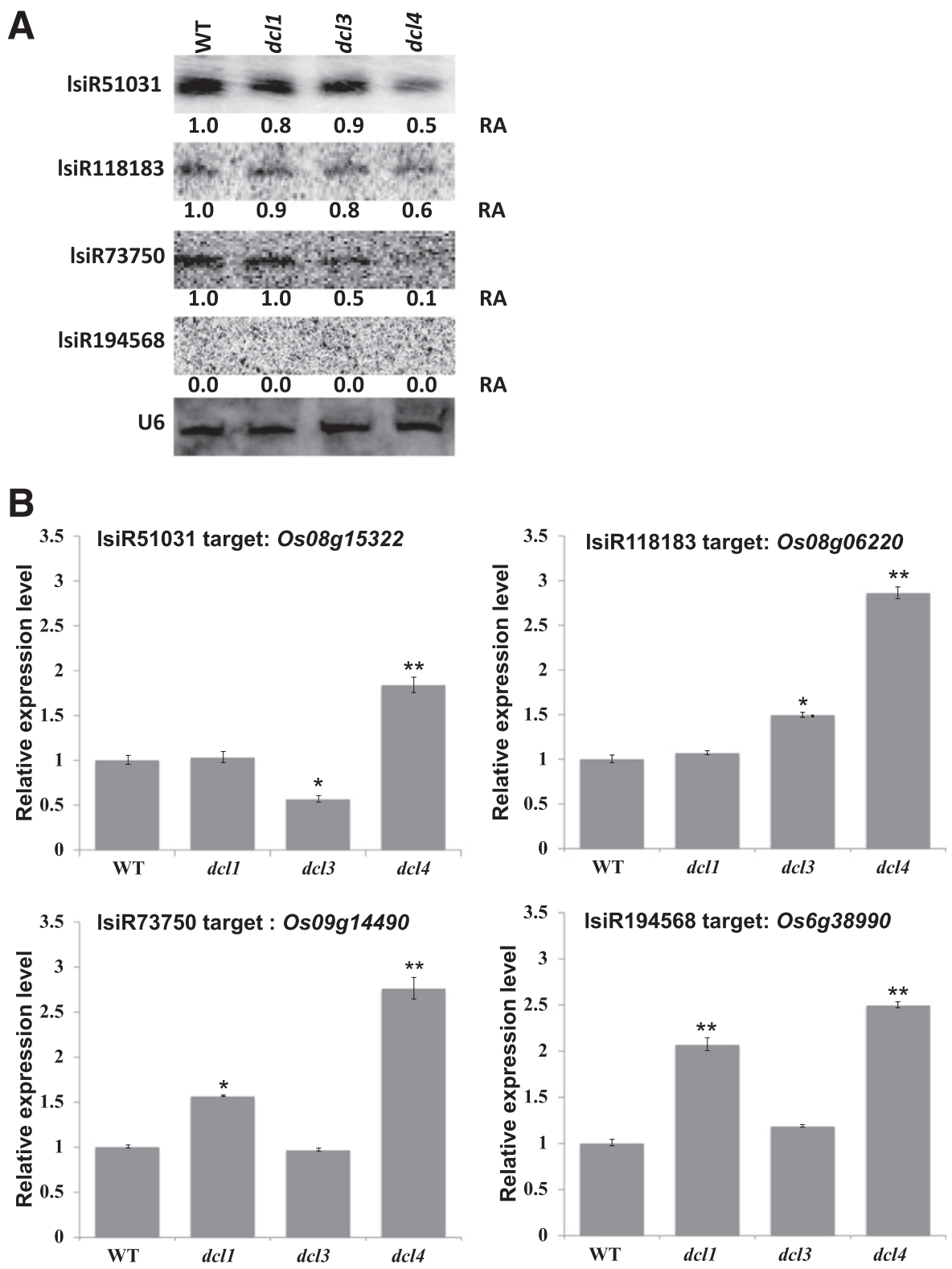

Fig. 4. Effects of $O s D C L s$ on the expression of lsiRNAs and their targets. A, Expression of osa-lsiRNAs dependents on OsDCL4. Accumulation of osa-lsiRNAs are detected with DNA oligonucleotides complementary to the indicated osa-lsiRNAs. U6 was used as the loading control. Similar results were obtained from two biological replicates. B, Expression of four osa-lsiRNAs target genes in $o s d c l$ healthy mutants is detected by real-time PCR. x-axis, different mutant plants; y-axis, relative expression of the detected target genes. Standard deviations were calculated from three technical replicates. $* * P<0.01$ and $* P<0.05$ (Student's $t$ test). Similar results were obtained from three biological replicates. 
infection (Lin et al. 2016). We hypothesized that if osa-lsiRNAs biogenesis and function follows a canonical pathway, that they might also be involved in plant defense response as well. In this study, we showed that rice endogenous lsiRNAs ( 25 to $40 \mathrm{nt}$ ) were expressed at measurable level and could suppress target gene expression, including some genes encoding defense-related proteins. We further demonstrated that the expression level of some osalsiRNAs were influenced by $R$. solani infection, which is facilitated by inhibited expression of defense-related genes.

lsiRNAs normally are overlooked for functional study due to their relative less prominent abundance (Jin and Zhu 2010; Voinnet 2009). Furthermore, these molecules sometimes are masked by spontaneous RNA degradation products, which makes it neglected by traditional investigation. Distribution analysis showed that osalsiRNAs identified in this study originated from various genome loci with sharp start and end positions (Supplementary Fig. S4). This is quite different from spontaneous RNA degradation, which shows stretched patterns along generating loci. In addition, northern detection showed that osa-lsiRNAs migrated as homogenous molecules, without trailing smears above or beneath them (Fig. 2A). These features distinguish osa-lsiRNAs from RNA degrades.

Higher plants possess many proteins with RNase III-type activity that are required for sRNA production. Dicer-like proteins (DCLs) are RNase III-type enzymes that cleave the double-stranded RNA precursors and process them into mature siRNAs. Rice encodes four types of DCLs with different functional specificities. OsDCL1 plays a critical role in miRNA processing in rice. Research showed that in rice mutant with $O s D C L 1$ defect, production of miRNA was severely reduced while the production of siRNA was not affected (Liu et al. 2005). OsDCL3 is involved in production of transposable element-associated 24-nt siRNAs and control certain agricultural traits in rice (Wei et al. 2014). OsDCL3 has two homologs, $O s D C L 3 a$ and $O s D C L 3 b$, the latter of which is associated with the processing of 24-nucleotide phased sRNA (phasiRNA) (Song et al. 2012). OsDCL4 is involved in processing of 21-nucleotide siRNAs, including transacting siRNAs (tasiRNA) and phasiRNA. By using mutants currently available to us (osdcll, osdcl3, and osdcl4), we were able to show that production of osa-lsiR51031, osa-lsiR73750,
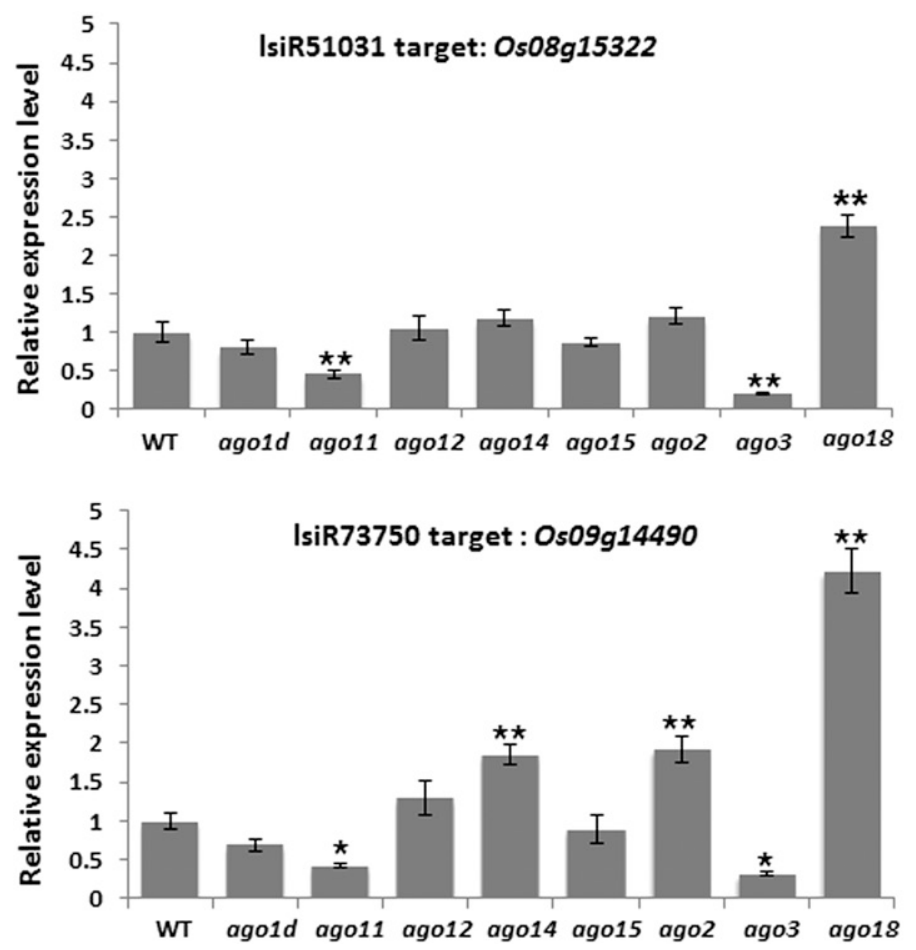

and osa-lsiR118183 requires OsDCLA. Osa-lsiR194568 was below detectable level and was not discussed here, as well as the $o s d c l 2$ mutant that is unavailable to us. These results suggest OsDCL4 plays an essential role in osa-lsiRNAs accumulation in rice. In Arabidopsis, generation of the 30- to 40-nt endogenous lsiRNAs requires $D C L 1$ and $D C L 4$ (Katiyar-Agarwal et al. 2007). Therefore, it seems that the involvement of DCLA in lsiRNA production is conserved between monocot and dicot plants.

In rice, phasiRNA is one type of siRNA whose production is dependent of OsDCL4. In osdcl4 loss-of-function mutants, dramatic reduction of phasiRNA was observed, regardless if it is the 21- or 24-nt type (Song et al. 2012). Therefore, we investigated whether osa-lsiRNAs are produced as phasiRNAs. Most of the 21and 24-nt phasiRNA clusters are initiated from the target sites of miR2118 or miR2275 (Song et al. 2012). Sequence analysis indicates that there are no target sites identified 2,000 nt up- or down-stream of the osa-lsiRNAs generating loci, indicating the generation of these osa-lsiRNAs is not triggered by cleavage of the 22-nt miRNAs such as miR2118 or miR2275. When the intermediate up- and down-stream regions were examined $(-2,000$ to $+2,000 \mathrm{nt}$ ), there was no observable siRNA in a phased pattern observed either, indicating these osa-lsiRNAs are not produced as phased siRNAs. Furthermore, the accumulation of the two triggering miRNAs (miR2118 or miR2275) requires OsDCL1 activity (Song et al. 2012). None of the osa-lsiRNAs required OsDCL1 for their biogenesis (Fig. 4A), further excluding the possibility that lsiRNAs are phasiRNAs.

Regardless of difference in biogenesis and mechanism of action, sRNAs need to be incorporate into the RNA-induced silencing complex (RISC) core by Argonaute proteins. So far about 19 rice Argonaute genes have been identified (Kapoor et al. 2008). According to phylogenetic relationships, plant Argonaute proteins can be divided into three major clades following the Arabidopsis AGO system (Fang and Qi 2016). The AGO1/5/10 clade includes $O s A G O 1,5,10,11,12,13,14$, and 17. OsAGO1 is associated with miRNA activity, defect of which causes pleiotropic developmental phenotypes (Wu et al. 2009). OsAGO5 is associated with 21-nt phasiRNA with a preference to siRNA started with a 5' C (Komiya
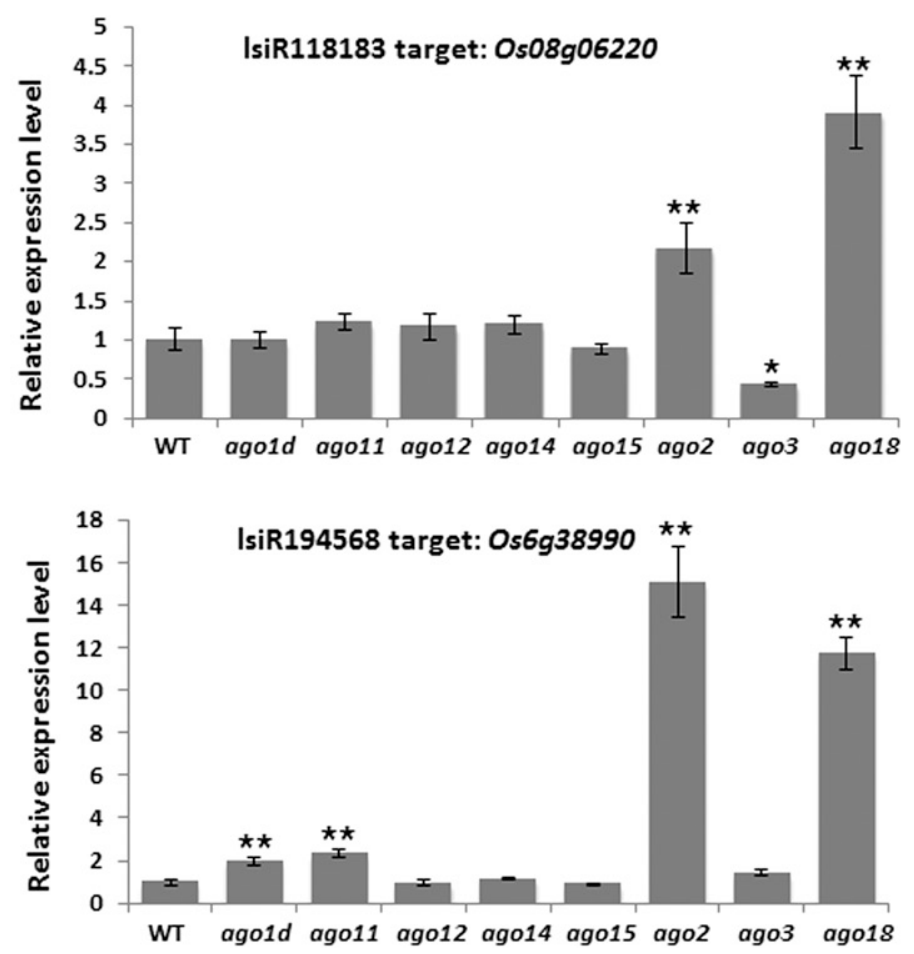

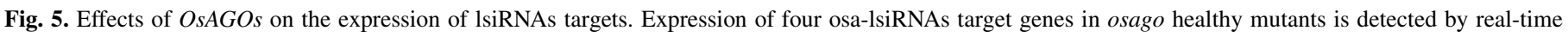

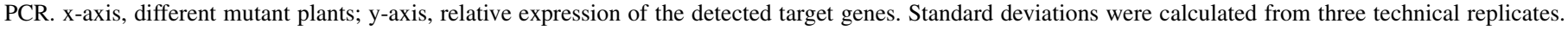
$* * P<0.01$ and $* P<0.05$ (Student's $t$ test). Similar results were obtained from three biological replicates. 
et al. 2014). OsAGO10 plays an important role in shoot apical meristem (SAM) maintenance, with specifically associated sRNA unidentified (Nishimura et al. 2002). In the osagold mutant plants we used, expression of target genes from each osa-lsiRNAs was examined by real-time PCR. Results showed that there was no discernable difference between the wild type and mutant plants, indicating OsAGOld is not involved in silencing of osa-lsiRNAs targets. We were not able to check other $A G O 1$ clade mutant unavailable to us. $\mathrm{Wu}$ and colleagues showed that rice encodes four $A G O 1$ homologs (AGOla, AGO1b, AGO1c, and AGO1d). AGOla, AGO1b, and $A G O 1 c$ all showed a strong preference for binding sRNAs with $5^{\prime} \mathrm{U}$ and have slicer activity. All three $A G O 1 s$ predominantly bound known miRNAs. Most of the miRNAs were evenly distributed in the three $A G O 1$ complexes (Wu et al. 2009). Taken together, we propose that $O s A G O 1$ is not required for osa-lsiRNAs silencing.

OsAGO2/3/7 form a unique clade, whose function is less reported. OsAGO7 is involved in tasiRNA biogenesis, some of which are involved in the regulation of SAM development (Nagasaki et al. 2007). AGO7 function is conserved in Arabidopsis, rice, and maize. Therefore, other components of the same clade might function similarly to their counterparts in Arabidopsis. In our study, we showed that the suppression of all the osa-lsiRNAs target genes was released in osago 2 mutant plants, indicating $O s A G O 2$ is required for osalsiRNAs silencing. In contrast, we did not observe any expression variation between osago3 and the wild-type plants, indicating $O s A G O 3$ is not involved in osa-lsiRNA-mediated gene silencing. This is in agreement with previous observation that although AGO2 and $A G O 3$ are close homologs from the same clade, their functions are different (Zhang et al. 2011).

Phylogenetically, $O s A G O 11,12$, and 14 are from the same clade as $A t A G O 4$. Rice encodes four $A G O 4$ homologs that are $O s A G O 4 a$, $b$, and OsAGO15 and 16 (Kapoor et al. 2008). OsAGO4a, b, and 16 can bind 24-nt siRNAs and lmiRNAs (Wu et al. 2010). Therefore, we tested whether osa-lsiRNA function is affected by rice plants defective in $A G O 4$ clade members. Our results indicated that none of the target expression was affected noticeably in these mutant plants (Fig. 5), indicating osa-lsiRNAs function through an alternate pathway without employing these AGOs.

$A G O 18$ is conserved in monocots, and has been characterized in rice and maize (Fang and Qi 2016). OsAGO18 is involved in resistance against viral infection. Defects in $O s A G O 18$ leads to increased susceptibility while its overexpression is associated with enhanced viral resistance (Wu et al. 2015). It was demonstrated that OsAGO18's contribution to viral defense is through preferential recruit of miR168, which liberates $O s A G O 1$ from miR168-mediated degradation and confer resistance function. Maize $A G O 18$ is associated with 24-nt phasiRNAs (Zhai et al. 2014). In osago18 plants, all the four target genes showed measurable increase, suggesting osa-lsiRNAs regulate their target gene expression through the OsAGO18 route.

Our results revealed that osa-lsiR51031 constitutively expressed in uninfected plants. After $R$. solani infection, the expression reduced dramatically (Fig. 2A). Osa-lsiR51031 targets a gene encoding cytochrome b559 subunit alpha (LOC_OsO8g15322), which is verified by transient co-expression in tobacco (Fig. 3). Cytochrome b559 is part of the reaction core of Photosystem II (PSII), which participate in an electron transport pathway that helps protect PSII from photo-damage (Chu and Chiu 2016). Moreover, Cytochrome b559 reveal an important role during temperature stress by rescuing PSII from oxidative damage (Tiwari et al. 2008). The expression of $O s 08 g 15322$ is induced by $R$. solani infection, which is in accordance with the decreasing expression of osalsiR51031 (Fig. 2B). The biological function of Cytochrome b559 in interaction between rice and $R$. solani, and the cause and consequence associated with induced Cytochrome b559 expression upon $R$. solani infection, is unknown yet.

Osa-lsiR118183 could target Os08g06220, which encodes a transferase. In rice, salicylic acid (SA) treatment can induce SA glucosyltransferase expression which is involved in plant immunity (Silverman et al. 1995). The homolog in Arabidopsis (At5g61160) is involved in the biosynthesis of hydroxycinnamic acid amides, which play a role in defense against pathogens (Muroi et al. 2009). Our results indicate that $R$. solani infection induces lsiR118183 expression, which leads to reduced expression of its target, the transferase. Therefore, $R$. solani infection may specifically reduce plant resistance by inhibiting expression of resistance-related genes through lsiRNA-mediated gene silencing. This hypothesis is verified by osa-lsiR194568 and osa-lsiR73750, which target a receptor-like protein kinase 2 (RLPK2) and a TIR-NBS type disease resistance protein (TNRP), respectively. Many studies have reported that RLPK and TNRP are involved in plant immunity. For example, Arabidopsis flagellin receptor FLS2, the EF-Tu receptor EFR (Gomez-Gomez and Boller 2000; Zipfel et al. 2006), RPS4 (Zhang et al. 2004), RPP1A (Weaver et al. 2006), and the rice protein PRR XA21 (Song et al. 1995) mediate immunity during plant-microbe interaction. Not surprisingly, both RLPK2 and TNRP were reduced upon $R$. solani infection, confirming our hypothesis that $R$. solani could manipulate plant innate immunity by inhibiting expression of key defense components.

In summary, our results revealed the existence of endogenous osa-lsiRNAs in rice, some of which are induced by $R$. solani infection. It seems that osa-lsiRNA-mediated gene silencing is employed by $R$. solani to break down host defense. Whether osa-lsiRNAs could manipulate host defense genes in response to other pathogens and how osa-lsiRNAs are affected by pathogen infection need further investigation.

Results for biological replicates are included in Supplementary Figure S5.

\section{ACKNOWLEDGMENTS}

This work was supported by the state key project (2016YFD0100602) to H.-W. Zhao; a National Natural Science Foundation of China (31501621) to D.-D. Niu and a grant from Natural Science Foundation of Jiangsu Province of China (BK20141360) to H.-W. Zhao.

\section{LITERATURE CITED}

Ambros, V., and Chen, X. 2007. The regulation of genes and genomes by small RNAs. Development 134:1635-1641.

Baulcombe, D. 2004. RNA silencing in plants. Nature 431:356-363.

Brown, J. D., and O’Neill, R. J. 2010. Chromosomes, conflict, and epigenetics: Chromosomal speciation revisited. Annu. Rev. Genomics Hum. Genet. 11: 291-316.

Carone, D. M., Longo, M. S., Ferreri, G. C., Hall, L., Harris, M., Shook, N., Bulazel, K. V., Carone, B. R., Obergfell, C., O’Neill, M. J., and O’Neill, R. J. 2009. A new class of retroviral and satellite encoded small RNAs emanates from mammalian centromeres. Chromosoma 118:113-125.

Chapman, E. J., and Carrington, J. C. 2007. Specialization and evolution of endogenous small RNA pathways. Nat. Rev. Genet. 8:884-896.

Chen, X. M. 2009. Small RNA metabolism and function in Arabidopsis. FASEB J. 23:194.1.

Chu, H. A., and Chiu, Y. F. 2016. The roles of cytochrome b (559) in assembly and photoprotection of photosystem II revealed by site-directed mutagenesis studies. Front. Plant Sci. 6:1261.

Duan, C. G., Wang, C. H., and Guo, H. S. 2012. Application of RNA silencing to plant disease resistance. Silence 3:5.

Fang, X., and Qi, Y. 2016. RNAi in plants: An Argonaute-centered view. Plant Cell 28:272-285.

Gomez-Gomez, L., and Boller, T. 2000. FLS2: An LRR receptor-like kinase involved in the perception of the bacterial elicitor flagellin in Arabidopsis. Mol. Cell 5:1003-1011.

Guo, W. X., Wu, G. T., Yan, F., Lu, Y. W., Zheng, H. Y., Lin, L., Chen, H. R., and Chen, J. P. 2012. Identification of novel Oryza sativa miRNAs in deep sequencing-based small RNA libraries of rice infected with Rice stripe virus. PLoS One 7:e46443.

Hock, J., and Meister, G. 2008. The Argonaute protein family. Genome Biol. 9:210.

Jin, H. L., and Zhu, J. K. 2010. How many ways are there to generate small RNAs? Mol. Cell 38:775-777.

Jones-Rhoades, M. W., Bartel, D. P., and Bartel, B. 2006. MicroRNAs and their regulatory roles in plants. Annu. Rev. Plant Biol. 57:19-53.

Kapoor, M., Arora, R., Lama, T., Nijhawan, A., Khurana, J. P., Tyagi, A. K., and Kapoor, S. 2008. Genome-wide identification, organization and 
phylogenetic analysis of Dicer-like, Argonaute and RNA-dependent RNA polymerase gene families and their expression analysis during reproductive development and stress in rice. BMC Genomics 9:451.

Katiyar-Agarwal, S., Gao, S., Vivian-Smith, A., and Jin, H. 2007. A novel class of bacteria-induced small RNAs in Arabidopsis. Genes Dev. 21: 3123-3134.

Katiyar-Agarwal, S., and Jin, H. L. 2010. Role of small RNAs in host-microbe interactions. Annu. Rev. Phytopathol. 48:225-246.

Komiya, R., Ohyanagi, H., Niihama, M., Watanabe, T., Nakano, M., Kurata, N., and Nonomura, K.-I. 2014. Rice germline-specific Argonaute MEL1 protein binds to phasiRNAs generated from more than 700 lincRNAs. Plant J. 78:385-397.

Lee, F. N., and Rush, M. C. 1983. Rice sheath blight: A major rice disease. Plant Dis. 67:829-832.

Li, Y., Lu, Y. G., Shi, Y., Wu, L., Xu, Y. J., Huang, F., Guo, X. Y., Zhang, Y., Fan, J., Zhao, J. Q., Zhang, H. Y., Xu, P. Z., Zhou, J. M., Wu, X. J., Wang, P. R., and Wang, W. M. 2014. Multiple rice MicroRNAs are involved in immunity against the blast fungus Magnaporthe oryzae. Plant Physiol. 164: 1077-1092.

Lin, H., and Spradling, A. C. 1997. A novel group of pumilio mutations affects the asymmetric division of germline stem cells in the Drosophila ovary. Development 124:2463-2476.

Lin, R., He, L., He, J., Qin, P., Wang, Y., Deng, Q., Yang, X., Li, S., Wang, S., Wang, W., Liu, H. N., Li, P., and Zheng, A. P. 2016. Comprehensive analysis of microRNA-Seq and target mRNAs of rice sheath blight pathogen provides new insights into pathogenic regulatory mechanisms. DNA Res. 23:415-425.

Liu, B., Li, P. C., Li, X., Liu, C. Y., Cao, S. Y., Chu, C. C., and Cao, X. F. 2005. Loss of function of OsDCL1 affects microRNA accumulation and causes developmental defects in rice. Plant Physiol. 139:296-305.

Muroi, A., Ishihara, A., Tanaka, C., Ishizuka, A., Takabayashi, J., Miyoshi, H., and Nishioka, T. 2009. Accumulation of hydroxycinnamic acid amides induced by pathogen infection and identification of agmatine coumaroyl transferase in Arabidopsis thaliana. Planta 230:517-527.

Nagasaki, H., Itoh, J.-I., Hayashi, K., Hibara, K.-I., Satoh-Nagasawa, N., Nosaka, M., Mukouhata, M., Ashikari, M., Kitano, H., Matsuoka, M., Nagato, Y., and Sato, Y. 2007. The small interfering RNA production pathway is required for shoot meristem initiation in rice. Proc. Natl. Acad. Sci. USA 104:14867-14871.

Naqvi, A. R., Haq, Q. M. R., and Mukherjee, S. K. 2010. MicroRNA profiling of Tomato leaf curl new delhi virus (tolcndv) infected tomato leaves indicates that deregulation of mir159/319 and mir172 might be linked with leaf curl disease. Virol. J. 7:281

Navarro, L., Dunoyer, P., Jay, F., Arnold, B., Dharmasiri, N., Estelle, M., Voinnet, O., and Jones, J. D. 2006. A plant miRNA contributes to antibacterial resistance by repressing auxin signaling. Science 312:436-439.

Nishimura, A., Ito, M., Kamiya, N., Sato, Y., and Matsuoka, M. 2002. OsPNH1 regulates leaf development and maintenance of the shoot apical meristem in rice. Plant J. 30:189-201.

Niu, D., Wang, Z., Wang, S., Qiao, L., and Zhao, H. 2015. Plant gene silencing: Methods and protocols. Pages 61-79 in: Profiling of Small RNAs Involved in Plant-Pathogen Interactions. K. S. Mysore and M. Senthil-Kumar, eds. Humana Press, New York.

Niu, D., Xia, J., Jiang, C., Qi, B., Ling, X., Lin, S., Zhang, W., Guo, J., Jin, H., and Zhao, H. 2016. Bacillus cereus AR156 primes induced systemic resistance by suppressing miR825/825* and activating defense-related genes in Arabidopsis. J. Integr. Plant Biol. 58:426-439.

Ouyang, S. Q., Park, G., Atamian, H. S., Han, C. S., Stajich, J. E., Kaloshian, I., and Borkovich, K. A. 2014. MicroRNAs suppress NB domain genes in tomato that confer resistance to Fusarium oxysporum. PLoS Pathog. 10: e1004464

Padmanabhan, C., Zhang, X., and Jin, H. 2009. Host small RNAs are big contributors to plant innate immunity. Curr. Opin. Plant Biol. 12:465-472.

Pumplin, N., and Voinnet, O. 2013. RNA silencing suppression by plant pathogens: Defence, counter-defence and counter-counter-defence. Nat. Rev. Microbiol. 11:745-760.

Rogers, K., and Chen, X. 2013. Biogenesis, turnover, and mode of action of plant microRNAs. Plant Cell 25:2383-2399.
Seo, J. K., Wu, J., Lii, Y., Li, Y., and Jin, H. 2013. Contribution of small RNA pathway components in plant immunity. Mol. Plant-Microbe Interact. 26: 617-625.

Silverman, P., Seskar, M., Kanter, D., Schweizer, P., Metraux, J. P., and Raskin, I. 1995. Salicylic-acid in rice-Biosynthesis, conjugation, and possible role. Plant Physiol. 108:633-639.

Song, W. Y., Wang, G. L., Chen, L. L., Kim, H. S., Pi, L. Y., Holsten, T., Gardner, J., Wang, B., Zhai, W. X., Zhu, L. H., Fauquet, C., and Ronald, P. 1995. A receptor kinase-like protein encoded by the rice disease resistance gene, Xa21. Science 270:1804-1806.

Song, X., Li, P., Zhai, J., Zhou, M., Ma, L., Liu, B., Jeong, D.-H., Nakano, M., Cao, S., Liu, C., Chu, C. C., Wang, X. J., Green, P. J., Meyers, B. C., and Cao, X. F. 2012. Roles of DCL4 and DCL3b in rice phased small RNA biogenesis. Plant J. 69:462-474.

Tiwari, A., Jajoo, A., and Bharti, S. 2008. Heat-induced changes in the EPR signal of tyrosine $\mathrm{D}\left(Y_{D}^{O X}\right)$ : A possible role of cytochrome b559. J. Bioenerg. Biomembr. 40:237-243.

Voinnet, O. 2009. Origin, biogenesis, and activity of plant microRNAs. Cell 136:669-687.

Weaver, L. M., Swiderski, M. R., Li, Y., and Jones, J. D. G. 2006. The Arabidopsis thaliana TIR-NB-LRR R-protein, RPP1A; Protein localization and constitutive activation of defense by truncated alleles in tobacco and Arabidopsis. Plant J. 47:829-840.

Wei, L., Gu, L., Song, X., Cui, X., Lu, Z., Zhou, M., Wang, L., Hu, F., Zhai, J., Meyers, B. C., and Cao, X. F. 2014. Dicer-like 3 produces transposable element-associated 24-nt siRNAs that control agricultural traits in rice. Proc. Natl. Acad. Sci. USA 111:3877-3882.

Wu, J., Yang, Z., Wang, Y., Zheng, L., Ye, R., Ji, Y., Zhao, S., Ji, S., Liu, R., Xu, L., Zheng, H., Zhou, Y. J., Zhang, X., Cao, X. F., Xie, L. H., Wu, Z. J., Qi, Y. J., and Li, Y. 2015. Viral-inducible Argonaute18 confers broadspectrum virus resistance in rice by sequestering a host microRNA. eLife 4:e05733.

Wu, L., Zhang, Q., Zhou, H., Ni, F., Wu, X., and Qi, Y. 2009. Rice microRNA effector complexes and targets. Plant Cell 21:3421-3435.

Wu, L., Zhou, H., Zhang, Q., Zhang, J., Ni, F., Liu, C., and Qi, Y. 2010. DNA methylation mediated by a microRNA pathway. Mol. Cell 38:465-475.

Xin, M., Wang, Y., Yao, Y., Xie, C., Peng, H., Ni, Z., and Sun, Q. 2010. Diverse set of microRNAs are responsive to powdery mildew infection and heat stress in wheat (Triticum aestivum L.). BMC Plant Biol. 10:123.

Ye, R., Chen, Z., Lian, B., Rowley, M. J., Xia, N., Chai, J., Li, Y., He, X. J., Wierzbicki, A. T., and Qi, Y. 2016. A Dicer-independent route for biogenesis of siRNAs that direct DNA methylation in Arabidopsis. Mol. Cell 61:222-235.

Zhai, J., Jeong, D. H., De Paoli, E., Park, S., Rosen, B. D., Li, Y., Gonzalez, A. J., Yan, Z., Kitto, S. L., Grusak, M. A., Jackson, S. A., Stacey, G., Cook, D. R., Green, P. J., Sherrier, D. J., and Meyers, B. C. 2011. MicroRNAs as master regulators of the plant NB-LRR defense gene family via the production of phased, trans-acting siRNAs. Genes Dev. 25:2540-2553.

Zhai, L., Sun, W., Zhang, K., Jia, H., Liu, L., Liu, Z., Teng, F., and Zhang, Z. 2014. Identification and characterization of Argonaute gene family and meiosis-enriched Argonaute during sporogenesis in maize. J. Integr. Plant Biol. 56:1042-1052.

Zhang, X., Zhao, H., Gao, S., Wang, W. C., Katiyar-Agarwal, S., Huang, H. D., Raikhel, N., and Jin, H. 2011. Arabidopsis Argonaute 2 regulates innate immunity via miRNA393(*)-mediated silencing of a Golgi-localized SNARE gene, MEMB12. Mol. Cell 42:356-366.

Zhang, Y., Dorey, S., Swiderski, M., and Jones, J. D. G. 2004. Expression of RPS4 in tobacco induces an AvrRps4-independent HR that requires EDS1, SGT1 and HSP90. Plant J. 40:213-224.

Zhao, H. W., Sun, R. B., Albrecht, U., Padmanabhan, C., Wang, A. R., Coffey, M. D., Girke, T., Wang, Z. H., Close, T. J., Roose, M., Yokomi, R. K., Folimonova, S., Vidalakis, G., Rouse, R., Bowman, K. D., and Jin, H. L. 2013. Small RNA profiling reveals phosphorus deficiency as a contributing factor in symptom expression for citrus huanglongbing disease. Mol. Plant 6:301-310.

Zipfel, C., Kunze, G., Chinchilla, D., Caniard, A., Jones, J. D. G., Boller, T., and Felix, G. 2006. Perception of the bacterial PAMP EF-Tu by the receptor EFR restricts Agrobacterium-mediated transformation. Cell 125:749-760. 